\title{
Effect of Size of Carbon Fiber on the Wear of PTFE Composites and Aluminum Alloy Counter Face
}

\author{
Agung Wibowo $^{1)}$, Yoshinori Takeichi ${ }^{1)^{*}}$, Takafumi Yamasaki ${ }^{1)}$ \\ Masahiro Kawamura $^{2)}$ and Masao Uemura ${ }^{1)}$ \\ ${ }^{1)}$ Department of Mechanical Engineering, Toyohashi University of Technology \\ 1-1 Hibarigaoka, Tempaku-cho, Toyohashi, Aichi 441-8580, Japan \\ ${ }^{2)}$ Kawamura Research Laboratories, Inc. \\ 1-5-6 Meguro, Meguro-ku, Tokyo 153-0063, Japan \\ *Corresponding author: takeichi@mech.tut.ac.jp
}

( Manuscript received 25 August 2008; accepted 17 January 2009; published 31 January 2009 )

\begin{abstract}
PTFE was reinforced with PAN-based, Pitch-based (carbonized and graphitized) carbon fibers and vapor-grown carbon fibers and their tribological properties were examined. Aluminum alloy was used as the counter face material. The wear rate of PTFE was only reduced by the vapor-grown carbon fiber and the composite filled with the vapor-grown carbon fiber caused minimum wear amount of the aluminum alloy counter face. Aluminum alloy was easily abraded by the carbon fibers other than vapor-grown carbon fibers, and then the abraded rough surface promoted the wear of PTFE composites. There was a correlation between the abrasiveness of carbon fiber and the cylindrical surface area of carbon fiber.
\end{abstract}

Keywords: PTFE, carbon fiber, abrasiveness, wear resistance, PAN-based carbon fiber, pitch-based carbon fiber, vapor-grown carbon fiber

\section{Introduction}

PTFE (polytetrafluoroethylene) is widely used as a tribomaterial by adding various kinds of fillers for the improvement of its tribological properties. Carbon fiber is one of the conventional filler materials to improve the mechanical strength and wear resistance of PTFE ${ }^{1)}$.

Lancaster $^{1)}$ studied the mechanism of wear reduction in polymers for carbon fiber reinforcement and proposed two factors. Firstly, the fibers were exposed at the sliding surface and supported part of the applied load; secondly, the fibers smoothed the surface of the counter face and thus reduced the localized stresses at the asperity contact.

Tanaka and Kawakami ${ }^{2)}$ studied the effects of the filler's shape and size on the wear resistance of PTFE composites and concluded that the load-supporting action and the prevention of large-scale destruction of the banded structures ${ }^{3)}$ of the PTFE matrix at frictional surfaces contribute to the wear-reducing action of the fillers.

The addition of carbon fibers is an effective method for reducing the wear amount of PTFE, but carbon fiber tends to abrade the counter face ${ }^{1)}$ and the abraded counter face induces the abrasion of PTFE composite.
Then it is possible that carbon fibers filled to suppress the wear of the PTFE composite promotes the wear of the PTFE composite.

Though the effects of the orientation of carbon fibers on the wear of the counter face have been studied ${ }^{1)}$, the effects of the size and type of carbon fibers on that and, inversely, the abrasiveness of the abraded counter face to the PTFE composites have not been reported extensively.

Then, the effects of the size and type of carbon fibers on the wear of PTFE composites and the counter faces were studied using several different types of carbon fibers. Relatively soft counter face metal was used in this study to enhance the counter face abrasion by the carbon fibers and to evaluate its effect on the wear amount quantitatively.

\section{Experiments}

2.1. Carbon fibers and sample preparation

Three kinds of PAN(polyacrylonitrile)-based carbon fibers, four kinds of pitch-based carbon fibers and a kind of vapor-grown carbon fiber were used as fillers to reinforce PTFE. Table 1 shows the length, diameter and mechanical properties of the carbon fibers obtained from the manufacturers' data sheets. The PAN-based 
carbon fiber, pitch-based carbon fibers and vapor-grown carbon fiber are labeled as "PAN-", "Pitch-" and "VGCF", respectively. For the PAN-based carbon fiber, "PAN-1" and "PAN-2" mean that they were produced by different manufacturers. The carbon fiber named "Pitch-C" and "Pitch-G" are the carbonized and graphitized pitch-based carbon fiber, respectively. The index of "S" and "L" added to the end of fiber name indicates relatively short and long fiber length, respectively.

Figure 1 shows the scanning electron microscopic (SEM) images of PAN-1S, Pitch-CS, Pitch-GS and VGCF. The surface of the pitch-based carbon fibers is smoother than that of PAN-based carbon fibers and there is no significant difference in the surface appearances between the carbonized and the graphitized carbon fiber.

A PTFE aqueous dispersion, Fluon AD911 (Asahi Glass Co., Ltd.), containing 60\% PTFE fine powder (median particle size of $0.25 \mu \mathrm{m}$ ) and one of the carbon fibers, 10 mass \% of PTFE fine powder, were mixed in a mortar machine for 50,000 times. During the mulling process, the liquid mixture of the PTFE dispersion and the carbon fiber was changed into a lump. The lump was cut into small pieces of about $5 \mathrm{~g}$ in weight and they were heated at $110{ }^{\circ} \mathrm{C}$ in the air for 90 minutes and the dried ones were pre-pressed into circular sheets. Then the sheet was heated in a vacuum of $3 \times 10^{-3} \mathrm{~Pa}$ at $380{ }^{\circ} \mathrm{C}$ for 2 hours and then pressed into a disk specimen of about $32 \mathrm{~mm}$ diameter at about $37 \mathrm{MPa}$ for 20 minutes at $380{ }^{\circ} \mathrm{C}$. The thickness of the disk specimen was about $2.5 \mathrm{~mm}$.

Table 1 Length, diameter and mechanical properties of carbon fibers used in the experiment

\begin{tabular}{|l|r|r|r|r|}
\hline $\begin{array}{c}\text { Carbon } \\
\text { fiber }\end{array}$ & $\begin{array}{c}\text { Length, } \\
\mu \mathrm{m}\end{array}$ & $\begin{array}{c}\text { Dia., } \\
\mu \mathrm{m}\end{array}$ & $\begin{array}{c}\text { Tensile } \\
\text { strength, } \\
\mathrm{MPa}\end{array}$ & $\begin{array}{c}\text { Tensile } \\
\text { modulus, } \\
\text { GPa }\end{array}$ \\
\hline PAN-1S & 30 & 7 & 3,530 & 230 \\
\hline PAN-1L & 150 & 7 & 3,530 & 230 \\
\hline PAN-2 & 40 & 7 & 3,920 & 235 \\
\hline Pitch-CS & 130 & 14.5 & 800 & 35 \\
\hline Pitch-CL & 3,000 & 14.5 & 800 & 35 \\
\hline Pitch-GS & 130 & 14.5 & 800 & 35 \\
\hline Pitch-GL & 3,000 & 14.5 & 800 & 35 \\
\hline VGCF & $10-20$ & 0.15 & & - \\
\hline
\end{tabular}

\subsection{Sliding test}

The configuration of sliding test was ring-on-disk. The disk specimen was carbon fiber filled PTFE composite prepared by the method mentioned above. The ring specimen was made of aluminum alloy (A5052, the Japanese Industrial Standards) and its Vickers hardness was about $55 \mathrm{HV}$. The outer diameter and inner diameter of the ring specimen were $16 \mathrm{~mm}$ and $12 \mathrm{~mm}$, respectively. The sliding surface of the ring specimen was polished to provide a surface roughness of less than

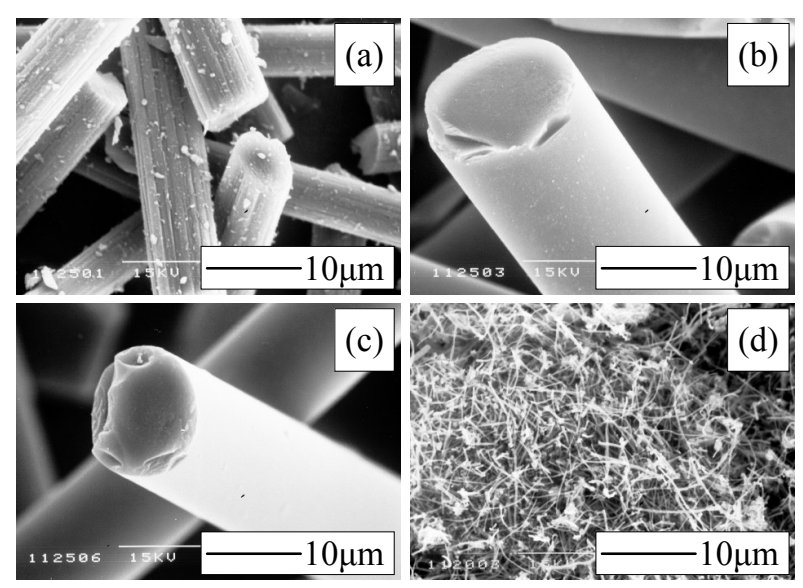

Fig. 1 SEM images of carbon fibers. (a) PAN-1S, (b) Pitch-CS, (c) Pitch-GS and (d) VGCF
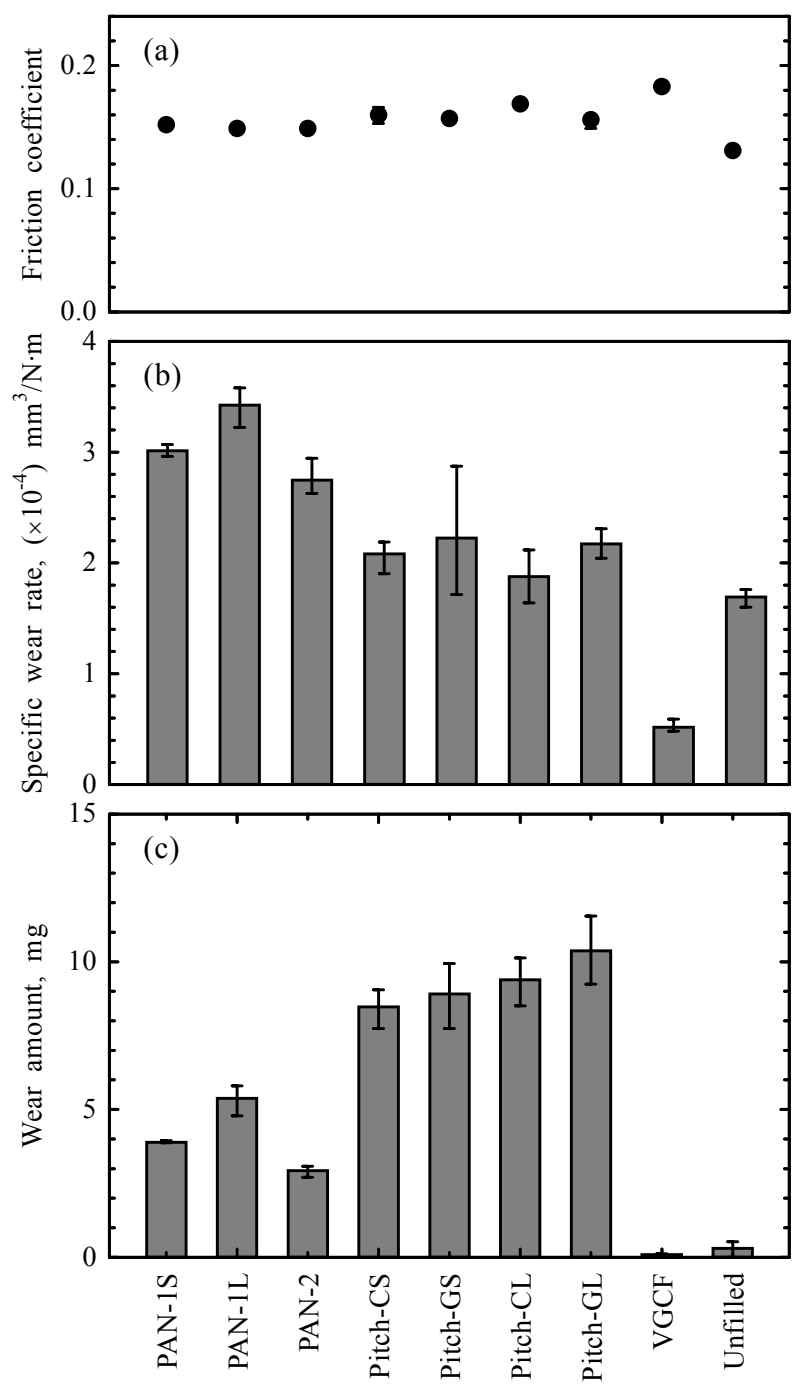

Fig. 2 (a) Friction coefficient, (b) specific wear rate of the disk specimen and (c) wear amount of the ring specimen

$0.03 \mu \mathrm{m}(R a)$. The ring specimens were cleaned ultrasonically with acetone before testing. The applied 
load was $490 \mathrm{~N}(5.6 \mathrm{MPa})$, the sliding speed was 0.22 $\mathrm{m} / \mathrm{s}$ and the sliding distance was $440 \mathrm{~m}$. The temperature of the atmospheric environment was room temperature. The relative humidity was controlled to be less than $30 \%$ by flowing dried air into the sliding test chamber. The sliding tests were conducted 3 times for one sort of disk specimens. The wear amount of the disk specimens was obtained by measuring the wear depth at 4 points on the wear track by a contact probe displacement sensor and then converted into wear volume by multiplying contact area. The wear amount of the ring specimens was obtained as a weight loss of the specimen by measuring the weight of each specimen before and after the sliding test.

\section{Results}

\subsection{Friction coefficient and wear amount}

The filled circles in Fig. 2(a) show the average friction coefficients and bars in Fig. 2(b) and 2(c) show the specific wear rates of disk specimens and the wear amounts of ring specimens. All composites are labeled as its filler name shown in table 1 and the disk specimen made from unfilled PTFE is labeled as Unfilled.

The wear rates of the disk specimens, except VGCF, were larger than that of the Unfilled as shown in Fig. 2(b). Thus the reinforcement of PTFE with conventional carbon fibers could not improve the wear resistance, when relatively soft aluminum alloy was used as the friction partner. On the other hand, the specific wear rate of VGCF whose friction coefficient had been the highest among the PTFE composites was 3 times lower than the Unfilled. The largest and the smallest wear amount of the ring specimen were obtained when they were rubbed against the PTFE composites filled with pitch-based carbon fiber and that filled with VGCF, respectively.

\subsection{Observations of friction tracks}

Figure 3 shows the typical scanning electron microscopic (SEM) images of wear tracks. Figure 3(a) is the wear track of the VGCF observed at a magnification of 1000. The fiber of VGCF was too thin to distinguish it from PTFE matrix at this magnification. Figure 3(b) is the wear track of its counter face. The transferred PTFE film was removed in patches. Figure 3 (c) is the wear track of PAN-1S. Many fibers are oriented toward sliding direction. Figure $3(d)$ is the wear track of its counter face. Patches and grooves seemed to be cut by the edge of fibers are observed. Figure 3(e) is the wear track of Pitch-GS. Fibers are oriented toward nearly sliding direction. Figure 3(f) is the wear track of its counter face. Many patches and grooves are also observed.

Figure 4 shows a series of profiles taken from the surface of the ring specimen before sliding and taken across the wear tracks on the ring specimen after sliding against PAN-1S, Pitch-CS, VGCF and Unfilled. Unfilled PTFE causes more damage to aluminum alloy

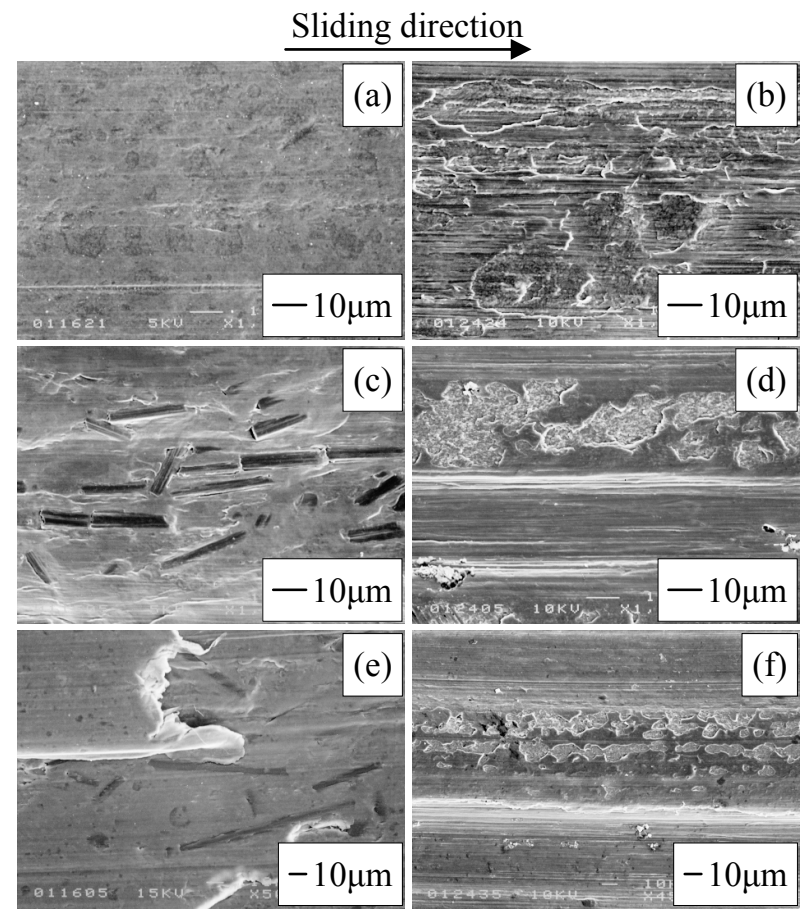

Fig. 3 SEM images of wear track. (a) / (b), (c) / (d) and (e) / (f) show the wear track of the disk specimen / the ring specimen for the VGCF, PAN-1S and Pitch-GS, respectively

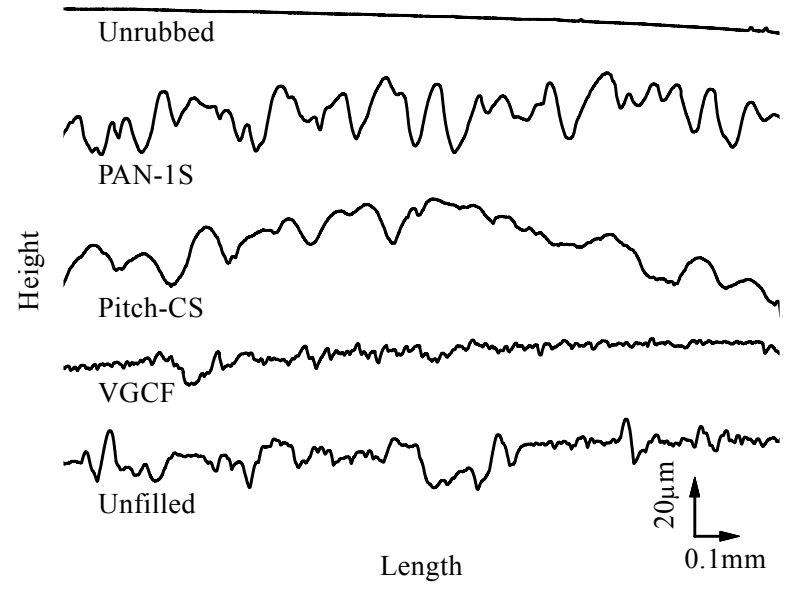

Fig. 4 Profiles taken from the surface of the aluminum alloy ring specimen before and after sliding test

than VGCF. Though the surface roughness of the wear track rubbed against PAN-1S and Pitch-CS are alike, the radius of asperity summits of the wear track rubbed against PAN-1S is appreciably smaller than that of the wear track rubbed against Pitch-CS.

\section{Discussion}

\subsection{Wear of ring specimen}

The abrasiveness of carbon fiber against the ring specimen increased according to the order of the diameter of the fiber, that is, the pitch-based carbon 
fiber was the most abrasive and the VGCF was the least abrasive. And when the diameter of the carbon fiber was the same, the longer carbon fiber was more abrasive than the shorter one.

As the contact area between a carbon fiber and PTFE matrix becomes large when the carbon fiber is long and thick, we can say that a long and thick carbon fiber can support larger reaction force that arise when the edge of the carbon fiber cuts the ring specimen's surface, than a short or thin one. So we obtained the relation between the wear amount of the ring specimen and the cylindrical surface area of the carbon fiber cylinder filled in the PTFE composite that was rubbed against the ring specimen.

There is a strong correlation between the wear amount of the ring specimen and the cylindrical surface area of the carbon fiber as shown in Fig. 5. This means that the aggression of carbon fibers against the counter face comes from the supporting capacity of the reaction force to cut the counter face with the edge of the carbon fiber. The degree of the effect to support the reaction force, however, seemed different between short fibers and long fibers as shown in Fig. 5. So we obtained the relation between the wear amount of the ring specimen and the cylindrical surface area of the carbon fiber using the wear data of 4 kinds of short carbon fibers, that is PAN-1S, PAN-2, Pitch-CS and Pitch-GS, by the least squares method.

The obtained equation is written by

$$
\log V=0.445 \log S-0.744
$$

Here $V$ is the wear amount of ring specimen and $S$ is the cylindrical surface area of the carbon fiber. The equation (1) is shown in Fig. 5 as a straight line.

By comparing the data points and the straight line, it is supposed that the values of the wear amounts for the ring specimens rubbed against the PTFE composites filled with Pitch-CL, Pitch-GL or VGCF shift toward the larger side of the carbon fiber's cylindrical surface area. As the direction of shifting suggests that long carbon fibers may have some surplus cylindrical surface area which have no effect on the aggression of the carbon fiber for the counter face, we calculate the effective cylindrical surface area for Pitch-CL and Pitch-GL by substituting the wear amounts of the ring specimens rubbed against Pitch-CL and Pitch-GL, that is $9.40 \mathrm{mg}$ for Pitch-CL and $10.38 \mathrm{mg}$ for Pitch-GL, into equation (1). Then we get $8125 \mu^{2}$ as the average cylindrical surface area. Because the diameter of Pitch-CL or Pitch-GL is $14.5 \mu \mathrm{m}$, the effective length of the carbon fiber is $178 \mu \mathrm{m}$. Then the effective length is $12.3(=178 / 14.5)$ times as long as the diameter of the fiber.

Using this multiplier, the effective length and the effective cylindrical surface area can be calculated as 7 $\times 12.3=86.1 \mu \mathrm{m}$ and $1,894 \mu \mathrm{m}^{2}$ for PAN-1L and $0.15 \times$ $12.3=1.85 \mu \mathrm{m}$ and $0.869 \mu \mathrm{m}^{2}$ for VGCF respectively. The relation between the wear amounts and the effective cylindrical surface area calculated are marked with crosses in Fig. 5. Because both of the crosses move toward the straight line, we can say there is an effective length for each fiber's diameter.

\subsection{Specific wear rate of carbon fiber filled PTFE compound}

Except the composite filled with VGCF, the specific wear rate of the composites was higher than unfilled PTFE. And the specific wear rate of the composites filled with PAN-based carbon fiber was higher than that of the composite filled with pitch-based carbon fiber, though the aggression for the counter face was the reverse.

Generally, it is known that the wear resistance of PTFE was improved with filling carbon fibers. Then it is possible that the decrease in the wear resistance was caused by the usage of the soft aluminum alloy as the counter face. The radius of asperity summits of the wear track on the ring specimen rubbed against PAN-1S is appreciably smaller than that of the wear track rubbed against Pitch-CS as shown in Fig. 4.

In case of abrasive wear, the wear mechanism ${ }^{1)}$ was supposed to be primarily one of penetration and ploughing of the hard asperities through the soft polymer. Then it is possible that the wear rate of the composite increased in the order of Pitch and PAN, because the radius of asperity summits of the counter face decreased in its order.

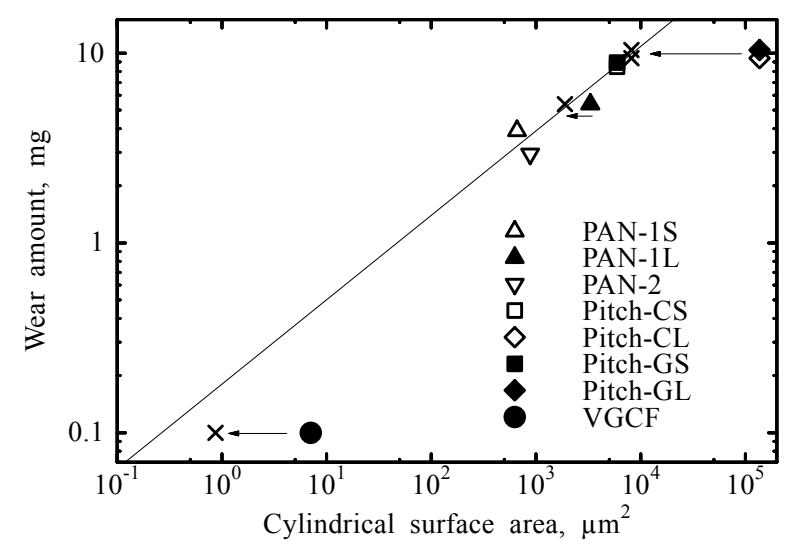

Fig. 5 The relation between the wear amount of the ring specimen and the cylindrical surface area of the carbon fibers

\section{Conclusions}

Tribological properties and abrasiveness of carbon fiber reinforced PTFE sliding against soft aluminum alloy were examined and the following conclusions were obtained.

(1) The vapor-grown carbon fibers only reduced the wear rate of PTFE, though the friction coefficient of the PTFE reinforced with those was higher than that of the unfilled PTFE and the PTFE reinforced with other carbon fibers. 
(2) The PTFE composites filled with PAN-based carbon fiber formed large and sharp asperities on the aluminum alloy counter face and showed the largest specific wear rate among tested PTFE composites.

(3) There was a correlation between the abrasiveness of carbon fibers and the size of their cylindrical surface area and the carbon fibers that had large cylindrical surface area were more abrasive than those that had small cylindrical surface area.

\section{Acknowledgements}

This work is carrying out supported by The Ministry of Education, Culture, Sports, Science and Technology (MEXT), Japan. Grant-in-Aid for Young Scientists (B): 18760111 .

\section{References}

[1] Lancaster, J. K., "The Effect of Carbon Fibre Reinforcement on the Friction and Wear of Polymers," J. Phys. D: Appl. Phys., 1, 1968, 549-559.

[2] Tanaka, K. and Kawakami, S., "Effect of Various Fillers on the Friction and Wear of Polytetrafluoroethylene-Based Composites," Wear 79, 1982, 221-234.

[3] Speerschneider, C. J. and Li, C. H., "Some Observations on the Structure of Polytetrafluoroethylene," J. Appl. Phys., 33, 5, 1962, 1871-1874. 